Pacific Journal of Mathematic 


\title{
EXTENDIBILITY, BOUNDEDNESS AND SEQUENTIAL CONVERGENCE IN SPACES OF HOLOMORPHIC FUNCTIONS
}

\author{
WILLIAM R. ZAME
}

\begin{abstract}
Let $X$ be a compact subset of $C^{m}$ and let $\mathscr{O}(X)$ be the space of germs on $X$ of functions holomorphic near $X$, equipped with its natural locally convex inductive limit topology. The object of this paper is to give, under a mild topological assumption on $X$, an internal description of this topology, and in particular, of the bounded sets and convergent sequences. These results follow from a general extendibility theorem. Surprisingly, the topological assumption on $X$ is necessary, and examples are constructed which illustrate this point. A related local extendibility result is also established.
\end{abstract}

The topology of $\mathscr{O}(X)$ may be described as follows. For each open set $U$ containing $X$, let $\mathscr{O}(U)$ denote the Frechet space of holomorphic functions on $U$, with the topology of uniform convergence on compact sets. Let $\rho_{U}: \mathcal{O}(U) \rightarrow \mathscr{O}(X)$ be the natural map. The space $\mathscr{O}(X)$ is the inductive limit of the spaces $\mathscr{O}(U)$, and we endow $\mathscr{O}(X)$ with the locally convex inductive limit topology; i.e., the finest locally convex topology which renders each of the maps $\rho_{U}$ continuous. Allan, Dales and McClure [2] have shown, using a general result of Komatsu [10], that this topology is in fact the finest (not necessarily locally convex) topology which renders the maps $\rho_{U}$ continuous. Thus, an arbitrary subset $\mathscr{F}$ of $\mathscr{O}(X)$ is closed if and only if $\rho_{U}^{-1}(\mathscr{F})$ is closed for each $U$. General functional-analytic results (see Edwards [5] for example) imply that $\mathscr{O}(X)$ is a complete, non-metrizable, locally convex space, and describe the bounded sets and convergent sequences in $\mathscr{O}(X)$. In particular, a subset $\mathscr{B}$ of $\mathscr{O}(X)$ is bounded if and only if there is an open set $U$ containing $X$ and a bounded set $\mathscr{B}_{U}$ in $\mathscr{O}(U)$ such that $\rho_{U}\left(\mathscr{B}_{U}\right)=\mathscr{B}$. Similarly, a sequence $f_{1}, f_{2} \ldots$ in $\mathscr{O}(X)$ converges to 0 if and only if there is an open set $U$ containing $X$ and a sequence $g_{1}, g_{2} \ldots$ in $\mathscr{O}(U)$ which converges to 0 , such that $\rho_{U}\left(g_{i}\right)=f_{i}$ for each $i$. It is then easy to see that a subset of $\mathscr{O}(X)$ is closed if and only if it is sequentially closed, so that despite its non-metrizability, the topology of $\mathcal{O}(X)$ is determined by its convergent sequences.

The above descriptions suffer from an unfortunate defect: they are not internal. That is, given a family $\mathscr{F}$ of germs in $\mathscr{O}(X)$, 
it is usually not clear whether there is a neighborhood of $X$ to which all the elements of $\mathscr{F}$ can be extended. The purpose of this note is to show that, under a very weak local connectedness assumption on $X$, it is possible to give internal conditions on $\mathscr{F}$ which are necessary and sufficient for the existence of an open set $U$ containing $X$ to which all the elements of $\mathscr{F}$ extend (Theorem 1). Easy applications of this result yield internal descriptions of the bounded sets in $\mathscr{O}(X)$ (Corollary 2) and the convergent sequences in $O(X)$ (Corollary 3). These descriptions are in terms of the rate of growth of successive derivatives of the functions in $\mathscr{F}$. We construct examples to show that the topological restrictions on $X$ cannot be removed (Theorem 4). Finally, we give a local version of our extendibility result (Theorem 5). The methods employed may be of interest in themselves and are largely topological.

The results in this paper answer a question raised by $R$. Aron at the conference on Infinite-Dimensional Holomorphy. The author would like to thank T. Hayden and T. Suffridge for their kind invitation to attend this Conference, and L. Mohler and D. Webster for several helpful conversations.

If $K, K^{\prime}$ are compact subsets of $X$ with $K \subset K^{\prime}$ then by $K^{\prime} / K$ we mean the space formed from $K^{\prime}$ by identifying $K$ to a point; we denote this point by $K / K$. We will say that $X$ has property $L$ if for each point $x$ in $X$ there is a finite sequence $K_{1} \subset K_{2} \subset \cdots \subset K_{n}$ of compact connected subsets of $X$ such that $K_{1}=\{x\}, K_{i+1} / K_{i}$ is locally connected for $i=1,2, \cdots, n-1$, and $X / K_{n}$ is locally connected at $K_{n} / K_{n}$. (For general information about point-set topology we refer to Whyburn [11]; we use Ahlfors and Sario [1] and Gunning and Rossi [7] as references for complex analysis.) That property $L$ is in fact a very weak form of local connectedness may be seen from the following example. Let $C_{1}$ be a Cantor set (i.e., a compact, totally disconnected perfect set) in the interval $\left\{(x, y) \in R^{2}: 0 \leqq x \leqq 1\right.$, $y=0\}$ which contains the point $(1,0)$ and let $C_{2}$ be a Cantor set in the interval $\left\{(x, y) \in R^{2}: 1 \leqq x \leqq 2, y=1\right\}$ which contains $(1,1)$. Let $X$ be the union of all straight-line intervals joining the point $(1,0)$ to a point of $C_{2}$ and all straight-line intervals joining the point $(1,1)$ to a point of $C_{1}$ Then $X$ has property $L$ but is not locally connected at any point.

Let $\mathscr{F}$ be a subset of $\mathscr{O}(X)$. We say that $\mathscr{F}$ is extendible if there is an open set $U$ containing $X$ and a family $\mathscr{F}_{U} \subset \mathcal{O}(U)$ for which $\rho_{U}\left(\mathscr{F}_{U}\right)=\mathscr{F}$. If $x \in X$, we say that the family $\mathscr{F}$ is continuable at $x$ if there is an open set $U_{x}$ containing $x$ such that for every $f$ in $\mathscr{F}$ there is a function $f^{x}$ in $\mathscr{O}\left(U_{x}\right)$ for which $f^{x}=f$ in some neighborhood of $x$. Note that $f^{x}$ is a continuation of $f$ into $U_{x}$, but need not represent an extension of $f$. Note also that 
continuability at $x$ is in fact an internal property, since it is equivalent to the requirement that the radii of convergence of the power series expansions (about $x$ ) of the elements of $\mathscr{F}$ are bounded away from 0 , and this latter requirement can be expressed, via the Hadamard radius formula, in terms of the values at $x$ of the elements of $\mathscr{F}$ and their derivatives. The following result is then an internal characterization of the extendible subsets of $\mathscr{O}(X)$ when $X$ has property $L$. Although we state the result only for compact subsets of $C^{m}$, it may be observed that the proof in fact carries through verbatim for compact sets in an analytic space, or in a complex manifold modelled on any metrizable topological vector space.

THEOREM 1. Let $X$ be a compact subset of $\boldsymbol{C}^{m}$ which has property $L$. Then a subset $\mathscr{F}$ of $\mathscr{O}(X)$ is extendible if and only if $\mathscr{F}$ is continuable at each point of $X$.

Proof. It is evident that an extendible family is continuable at each point, so we need to establish the converse. Note first of all that there is no loss in assuming the family $\mathscr{F}$ to be countable; say $\mathscr{F}=\left\{f_{1}, f_{2}, \cdots\right\}$. We will proceed by constructing a complex manifold $\Sigma$ on which all the functions in $\mathscr{F}$ "live", and then show that $\Sigma$ contains a copy of $X$. We will then "push down" a neighborhood of $X$ in $\Sigma$ to obtain the desired extension in $C^{m}$.

For each $x$ in $X$, let $U_{x}$ be the neighborhood of $x$ provided by the definition of continuability at $x$, and let $f_{j}^{x}$ be the continuation of $f_{j}$ into $U_{x}$. Set

$$
\Lambda=\left\{(x, a): x \in X, a \in U_{x}\right\}
$$

Note that $\Lambda$ is the disjoint union of the collection $\left\{U_{x}\right\}$ and thus may be given the structure of a complex-analytic manifold (with uncountably many connected components). Let $p: \Lambda \rightarrow C^{m}$ be defined by $p(x, a)=a ; p$ is easily seen to be a holomorphic local homeomorphism.

For each integer $n$, define an equivalence relation $\mathscr{R}_{n}$ on $\Lambda$ by requiring that $(x, a) \mathscr{R}_{n}(y, b)$ if $a=b$ and $f_{j}^{x}=f_{j}^{y}$ in a neighborhood of the point $a=b$, for each $j=1,2, \cdots, n$. Let $\Sigma_{n}$ be the quotient space of $\Lambda$ with respect to this equivalence relation, with the quotient topology. We have the quotient map $\sigma_{n}: \Lambda \rightarrow \Sigma_{n}$ and an induced map $\tau_{n}: \Sigma_{n} \rightarrow C^{m}$ which are easily seen to be local homeomorphisms with $\tau_{n} \circ \sigma_{n}=p$. Thus $\tau_{n}$ induces on $\Sigma_{n}$ the structure of a complex-analytic manifold and $\sigma_{n}, \tau_{n}$ are holomorphic maps. Finally let $\mathscr{R}$ be the equivalence relation on $A$ defined by $(x, a) \mathscr{R}(y, b)$ 
if $(x, a) \mathscr{R}_{n}(y, b)$ for all $n$. Let $\Sigma$ be the quotient space of $\Lambda$ with respect to $\mathscr{R}$; as above we obtain local homeomorphisms $\sigma: \Lambda \rightarrow \Sigma$ and $\tau: \Sigma \rightarrow C^{m}$ such that $\tau \circ \sigma=p$. We also obtain natural maps $\zeta_{n}: \Sigma \rightarrow \Sigma_{n}$ and $\psi_{n}: \Sigma_{n+1} \rightarrow \Sigma_{n}$. When we equip $\Sigma$ with the complex structure induced by $\tau$, we arrive at the commutative diagram of complex-analytic manifolds and holomorphic local homeomorphisms shown in the figure. This completes the construction of the desired complex-analytic manifold $\Sigma$.

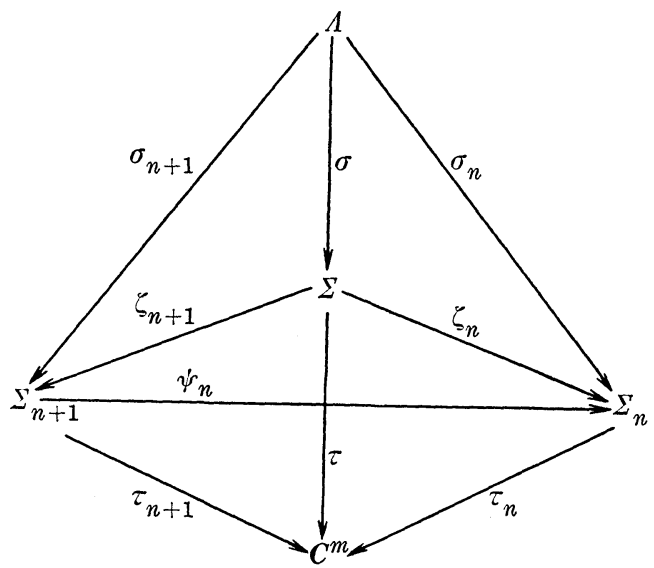

FIGURE

Now we show that $\Sigma$ contains a copy of $X$. Define $\varphi: X \rightarrow \Lambda$ by $\varphi(x)=(x, x)$. We wish to show that $\sigma \circ \varnothing$ is a homeomorphism of $X$ into $\Sigma$; it is clearly one-to-one, so we need to establish its continuity. For each $x$ in $X$ and each integer $j$, the functions $f_{j}$ and $f_{j}^{x}$ have the same germ at $x$, and hence have the same germ at all points $y$ in some $X$-neighborhood of $x$. Hence for each $x$ in $X$ and each integer $n$ there is an $X$-neighborhood $V$ of $x$ for which $f_{j}$ and $f_{j}^{x}$ have the same germ at $y$ for each $y$ in $V$ and each $j=$ $1,2, \cdots, n$. It follows easily that $\sigma_{n} \circ \varphi=\zeta_{n} \circ \sigma \circ \phi$ is continuous for each $n$. If $\Sigma$ were the inverse limit of the spaces $\Sigma_{n}$ we would then have the continuity of $\sigma \circ \varphi$; unfortunately, $\Sigma$ does not carry the inverse limit topology, so we use a different procedure.

We will proceed by establishing the following principle: if $K, K^{\prime}$ are compact, connected subsets of $X$ such that $K \subset K^{\prime}, K^{\prime} / K$ is locally connected at $K / K$ and $(\sigma \circ \varphi) \mid K$ is continuous, then $(\sigma \circ \varphi) \mid K^{\prime}$ is continuous at each point of $K$. Note first that $\sigma \circ \varphi(K)$ is a compact connected subset of $\Sigma$ on which $\tau$ is one-to-one. Since $\tau$ is a local homeomorphism, a compactness argument allows us to find a connected neighborhood $V$ of $\sigma \circ \varphi(K)$ in $\Sigma$ such that $\tau \mid V$ is a homeomorphism onto the open set $\tau(V) \subset C^{m}$. Let $W$ be the connected 
component of $\tau(V) \cap K^{\prime}$ which contains $K$; we claim that $\sigma \circ \varphi(W) \subset$ $V$. If this were not so, we could find a point $w$ in $W$ with $\sigma \circ \varphi(w) \notin V$ and a point $v$ in $V$ for which $\tau(v)=w$. It is evident that the maps $\zeta_{n}$ collectively distinguish the points of $\Sigma$, so we can find an index $k$ for which $\zeta_{k}(w) \neq \zeta_{k}(v)$. Note that $\zeta_{k} \mid V$ is one-to-one since $\tau \mid V$ is one-to-one. Then if we set $T=\zeta_{k} \circ \sigma \circ \varphi(W)$, we see that $T$ contains $\zeta_{k} \circ \sigma \circ \varphi(K)$, which is a subset of $\zeta_{k}(V)$, and also contains $\zeta_{k}(w)$ which is a point not in $\zeta_{k}(V)$. Since $\zeta_{k} \circ \sigma \circ \phi$ is continuous on $X, T$ is a connected set. On the other hand, $\tau_{k}$ is a local homeomorphism and $\tau_{k} \mid \zeta_{k}(V)$ is easily seen to be a homeomorphism onto $\tau(V)$, so that $\zeta_{k}(V)$ must be a connected component of $\tau_{k}^{-1}(\tau(V))$. Since $T$ is a connected subset of $\tau_{k}^{-1}(\tau(V))$ and meets $\zeta_{k}(V)$ it follows that $T \subset \zeta_{k}(V)$. This contradiction establishes our claim that $\sigma \circ \phi(W) \subset V$. Now, $\tau \mid V$ is a homeomorphism onto $\tau(V)$, so $\tau \mid \sigma \circ \varphi(W)$ is a homeomorphism onto $W$, and $\sigma \circ \varphi \mid W$ is its inverse and is therefore continuous. Since $K^{\prime} / K$ is locally connected at $K / K$, it follows that $W$ is a neighborhood of $K$ in $K^{\prime}$, so that $(\sigma \circ \varphi) \mid K^{\prime}$ is continuous at each point of $K$, which establishes the desired principle.

To see that $\sigma \circ \phi$ is in fact continuous on $X$, let $x$ be in $X$ and let $K_{1}, K_{2}, \cdots, K_{n}$ be the sequence of compact connected sets whose existence is guaranteed by the definition of property $L$. Since $K_{2}$ is locally connected and $\sigma \circ \phi \mid\{y\}$ is continuous for each $y$ in $K_{2}$, application of the above principle to each of the pairs $\{y\}, K_{2}$ shows that $\sigma \circ \varphi \mid K_{2}$ is continuous. Since $K_{3} / K_{2}$ is locally connected, application of the principle to the pair $K_{2}, K_{3}$ and then to each of the pairs $\{z\}, K_{3}$ for each $z$ in $K_{3} \backslash K_{2}$ yields the continuity of $\sigma \circ \varphi \mid K_{3}$. Continuing, we see that $\sigma \circ \varnothing \mid K_{n}$ is continuous. Finally, application of the principle to the pair $K_{n}, X$ shows that $\sigma \circ \varphi$ is continuous at each point of $K_{n}$, and in particular at $x$. Since $x$ was arbitrary, it follows that $\sigma \circ \varphi$ is a homeomorphism, as desired.

For each $j$, define a function $\widetilde{f}_{j}$ on $\Sigma$ by $\widetilde{f}_{j}(\sigma(x, a))=f_{j}^{x}(a)$. It is easily checked that $\widetilde{f}_{j}$ is in fact well-defined and holomorphic on $\Sigma$, and that $\widetilde{f}_{j}(\sigma(x, x))=f_{j}(x)$. Since $\sigma \circ \varphi(X)$ is a compact set in $\Sigma$ and $\tau \mid \sigma \circ \varphi(X)$ is a homeomorphism, there is a neighborhood $Q$ of $\sigma \circ \varphi(X)$ in $\Sigma$ such that $\tau \mid Q$ is a homeomorphism of $Q$ onto the open set $\tau(Q) \subset C^{m}$. For each $j$, define a function $g_{j}$ on $\tau(Q)$ by $g_{j}=$ $\tilde{f}_{j} \circ(\tau \mid Q)^{-1}$. It is easy to see that $g_{j}$ is a holomorphic extension of $f_{j}$ to the open set $\tau(Q)$ containing $X$, so that the family $\mathscr{F}$ is indeed extendible.

With the aid of this extendibility result, we can easily establish the desired internal descriptions of the bounded sets and covergent sequences in $O(X)$.

Corollary 2. Let $X$ be a compact subset of $C^{m}$ which has 
property $L$ and let $\mathscr{F}$ be a subset of $\mathscr{O}(X)$. Then $\mathscr{F}$ is bounded if and only if for each $x$ in $X$ there is a constant $M$ such that

$$
\left|\frac{\partial^{k_{1}+\cdots+k_{m}} f}{\partial z_{1}^{k_{1}} \cdots \partial z_{m}^{k_{m}}}(x)\right| \leqq\left(k_{1} ! \cdots k_{m} !\right) M^{k_{1}+\cdots+k_{m}}
$$

for each $f$ in $\mathscr{F}$ and each $k_{1}, k_{2}, \cdots, k_{m}$.

Proof. That the bounded sets in $\mathscr{O}(X)$ have this property follows easily from an application of the Cauchy integral formula. To establish the converse, we use the Hadamard radius formula to conclude that $\mathscr{F}$ is continuable at each point of $X$. Theorem 1 allows us to conclude that there is an open set $U$ containing $X$ and a family $\mathscr{F}_{U} \subset \mathscr{O}(U)$ for which $\rho_{U}\left(\mathscr{F}_{U}\right)=\mathscr{F}$. For each $x$ in $X$, choose a polydisk $D_{x}$ centered at $x$ and contained in $U$. Condition (B) combined with a straightforward estimate using power series shows that $\mathscr{F}_{U} \mid D_{x}$ is bounded in $\mathcal{O}\left(D_{x}\right)$. Since $X$ is compact, we can choose a finite number of such polydisks $D_{x_{1}}, D_{x_{2}}, \cdots, D_{x_{n}}$, which cover $X$. Set $D=\bigcup D_{x_{i}}$; then $\mathscr{F} \mid W$ is a bounded set in $\mathscr{O}(W)$, which is the desired result.

The proof of the following result requires only a slight modification of the above and is omitted.

Corollary 3. Let $X$ be a compact subset of $C^{m}$ which has property $L$. Then the sequence $f_{1}, f_{2}, \cdots$ converges to 0 in $O(X)$ if and only if for each $x$ in $X$ there is a constant $M$ with the property that for every $\varepsilon>0$ there is an integer $N_{\varepsilon}$ such that

$$
\left|\frac{\partial^{k_{1}+\cdots+k_{m}} f_{j}}{\partial z_{1}^{k_{1}} \cdots \partial z_{m}^{k_{m}}}(x)\right| \leqq \varepsilon\left(k_{1} ! \cdots k_{m} !\right) M^{k_{1}+\cdots+k_{m}}
$$

for every $k_{1}, \cdots, k_{m}$ and every $j \geqq N_{\varepsilon}$.

We remark that certain topological assumptions on $X$, other than property $L$, would suffice for the above result. For example, we could assume the existence of compact connected sets $J_{1}, J_{2}, \ldots, J_{n}$ with $J_{1} \subset J_{2} \subset \cdots \subset J_{n}=X, J_{1}$ locally connected and $J_{i+1} / J_{i}$ locally connected for each $i=1,2, \cdots, n-1$. It is not hard to see that this assumption is not implied by (nor does it imply) property $L$.

It is easy to see that Theorem 1 and Corollaries 2 and 3 are false without some sort of topological restriction on $X$. Suppose for example, that $X$ has infinitely many connected components. Then at least one of them, say $X_{0}$, is not an open and closed subset of $X$. Choose a sequence $V_{1}, V_{2}, \ldots$ of neighborhoods of $X_{0}$ whose boundaries do not intersect $X$ such that $X_{0}=\bigcap V_{i}$. Let $g_{k}$ be the 
function which is $2^{-k}$ on $V_{k}$ and 0 on $C^{m}-\bar{V}_{k}$. Then $\left\{g_{k}\right\}$ is a sequence in $\mathcal{O}(X)$ which is continuable at each point of $X$, satisfies conditions (B) and (C) of Corollaries 2 and 3 respectively, but is not extendible (and thus neither bounded nor convergent). Construction of a counter-example in which $X$ is connected is much more difficult, but is accomplished in the following Theorem.

THEOREM 4. There is a compact connected subset $X$ of $C^{2}$ and a countable set in $\mathscr{O}(X)$ which is continuable at each point of $X$ but not extendible.

Proof. Let $U_{0}=\left\{(z, w) \in C^{2}: z \neq 0\right\}$ and let $x=(1,1)$. Using an inductive procedure, we can construct a sequence $U_{1}, U_{2}, \ldots$ of connected open sets containing $x$ with the following properties:

(i ) $U_{j+1}$ is a relatively compact subset of $U_{j}$;

(ii) if $y \in U_{j+1}$ then the distance from $y$ to the boundary of $U_{j+1}$ does not exceed $2^{-j}$;

(iii) $U_{j+1}$ is an open solid torus which "winds around" $U_{j}$ exactly twice.

(This is simply the procedure for constructing a dyadic solenoid. A detailed geometric construction may be found by [4, pp. 70-72].) Condition (iii) insures two things: First, that $\pi_{1}\left(U_{j}, x\right)$ (the fundamental group of $U_{j}$ with base point $x$ ) is just $Z$ for each $j=0,1, \ldots$; and second, that the inclusion $U_{j+1} \rightarrow U_{j}$ induces a monomorphism $\pi_{1}\left(U_{j+1}, x\right) \rightarrow \pi_{1}\left(U_{0}, x\right)$ whose image is the subgroup $2^{j} Z$. Let $X=\bigcap U_{j}$; then $X$ is a compact, connected subset of $C^{2}$ which contains $x$ (in fact $X$ is a dyadic solenoid).

For each $k=1,2, \cdots$ let $\varphi_{k}: U_{0} \rightarrow U_{0}$ be given by $\varphi_{k}(z, w)=$ $\left(z^{2^{k}}, w\right)$. Then each $\varphi_{k}$ is a covering map and $\varphi_{k}$ induces a homomorphism $\varphi_{k^{*}}: \pi_{1}\left(U_{0}, x\right) \rightarrow \pi_{1}\left(U_{0}, x\right)$ whose image is the subgroup $2^{k} Z$. By the general theory of covering spaces (see [6] for example) there is a unique map $\psi_{k}: U_{k} \rightarrow U_{0}$ such that $\varphi_{k} \circ \psi_{k}$ is the inclusion of $U_{k}$ in $U_{0}$ and $\psi_{k}(x)=x$. Evidently $\psi_{k}$ is a homeomorphism, and is holomorphic (since $\varphi_{k}$ is).

For each $k=1,2, \cdots$ define a function $f_{k}$ on $U_{k}$ by $f_{k}=z \circ \psi_{k}$. Thus $f_{k}$ is a holomorphic branch of the function $z^{2^{-k}}$. We assert that for every $k \geqq 2, f_{k}$ has no extension to $U_{k-1}$. This can be seen by a direct and very messy argument, but we present an alternative method. Suppose that $g$ were such an extension. Let $O$ be the sheaf of germs of holomorphic functions on $C^{2}$, and let $\nu: \mathcal{O} \rightarrow C^{2}$ be the projection. Define a map $\gamma: U_{0} \rightarrow \mathcal{O}$ as follows. For each $t$ in $U_{0}$, let $W_{t}$ be an open neighborhood of $t$ such $\varphi_{k} \mid W_{t}$ is one-to-one, and set $h=z \circ\left(\varphi_{k} \mid W_{t}\right)^{-1}$. Let $\gamma(t)$ be the germ of $h$ at $\varphi_{k}(t)$. A simple computation shows that $\gamma$ is well-defined and a 
homeomorphism; moreover, $\nu \circ \gamma=\varphi_{k}$ so that

$$
\nu \mid \gamma\left(U_{0}\right): \gamma\left(U_{0}\right) \longrightarrow U_{0}
$$

is a covering map. Now define a map $\eta: U_{k-1} \rightarrow \infty$ by sending $t$ to the germ of $g$ at $t ; \eta$ is a homeomorphism onto its range, which is open. Since $x=(1,1), \varphi_{k}(x)=x$ and $\eta(x)=\gamma(x)$. We claim that $\eta\left(U_{k-1}\right) \subset \gamma\left(U_{0}\right)$; since $\eta\left(U_{k-1}\right)$ is a connected subset of $\nu^{-1}\left(U_{0}\right)$ which meets $\gamma\left(U_{0}\right)$ (by the above), it will suffice to show that $\gamma\left(U_{0}\right)$ is an open and closed subset of $\nu^{-1}\left(U_{0}\right)$. It is certainly open, so suppose that $\alpha$ is a point of its boundary in $\nu^{-1}\left(U_{0}\right)$. Recall that $\nu$ is a local homeomorphism, so that $\nu^{-1}(\nu(\alpha))$ is discrete, and that $\nu \mid \gamma\left(U_{0}\right)$ is a covering map. Hence we can find a connected neighborhood $W_{\alpha}$ of $\alpha$ in $\nu^{-1}\left(U_{0}\right)$ and, for each $\beta$ in $\gamma\left(U_{0}\right) \cap \nu^{-1}(\nu(\alpha))$, a connected neighborhood $W_{\beta}$ of $\beta$ in $\gamma\left(U_{0}\right)$ such that: (a) $W_{\alpha} \cap W_{\beta}=\phi=W_{\beta} \cap W_{\beta^{\prime}}$ for all $\beta, \beta^{\prime}$ in $\gamma\left(U_{0}\right) \cap \nu^{-1}\left(\nu(\alpha)\right.$ ) with $\beta \neq \beta^{\prime}$; (b) $\nu\left(W_{\alpha}\right)=\nu\left(W_{\beta}\right)$ is an open neighborhood of $\nu(\alpha)$ which is evenly covered by $\nu \mid \gamma\left(U_{0}\right)$. Hence $\nu$ maps each connected component of $\nu^{-1}\left(\nu\left(W_{\alpha}\right)\right) \cap \gamma\left(U_{0}\right)$ onto $\nu\left(W_{\alpha}\right)$. But our construction insures that at least one of these components is contained in $W_{\alpha} \cap \gamma\left(U_{0}\right)$ and hence contains no point of $\nu^{-1}(\alpha)$. This contradiction allows us to conclude that $\eta\left(U_{k-1}\right) \subset \gamma\left(U_{0}\right)$ as claimed. Now, if $\iota: U_{k-1} \rightarrow U_{0}$ is the inclusion then $\left(\nu \mid \gamma\left(U_{0}\right)\right) \circ \eta=\ell$, so that

$$
\left(\nu \mid \gamma\left(U_{0}\right)\right)_{*} \circ \eta_{*}=\iota_{*}: \pi_{1}\left(U_{k-1}, x\right) \longrightarrow \pi_{1}\left(U_{0}, x\right) .
$$

On the other hand, the range of $\iota_{*}$ is the subgroup $2^{k-1} Z$, while the range of $\left(\nu \mid \gamma\left(U_{0}\right)\right)_{*}$ is the range of $\left(\varphi_{k}\right)_{*}$ (since $\gamma$ is a homeomorphism and $\left(\nu \mid \gamma\left(U_{0}\right)\right) \circ \gamma=\varphi_{k}$ ) which is the subgroup $2^{k} Z$ This contradiction establishes our claim that $f_{k}$ has no extension to $U_{k-1}$.

Now the family $\left\{f_{k}\right\}$ in $\mathcal{O}(X)$ is evidently not extendible, but since each $f_{k}$ is a root of the function $z$ and $X$ is a compact set disjoint from $\{(z, w): z=0\}$, the family $\left\{f_{k}\right\}$ is indeed continuable at each point of $X$. We remark that the family $\left\{f_{k}\right\}$ actually satisfies condition (B) of Corollary 2.

There is a local notion of extendibility which is relevant here. We say that a family $\mathscr{F} \subset \mathcal{O}(X)$ is extendible at the point $x$ in $X$ if there is a compact neighborhood $D_{x}$ of $x$ such that every element of $\mathscr{F}$ extends to a neighborhood of $X \cup D_{x}$. It is perhaps not evident that a family which is extendible at each point of $X$ is in fact extendible, but this is indeed the case. To prove this, we need only carry out the argument of Theorem 1, and observe that the need for property $L$ is vitiated since the functions in question are assumed to extend to $X \cup D_{x}$ (rather than merely continuing). In light of this, the following local result is somewhat surprising. 
Theorem 5. Let $X$ be a compact subset of $C$ and let $x$ be a point of $X$. The following conditions are equivalent:

(i) $X$ is locally connected at $x$;

(ii) every family in $\mathcal{O}(X)$ which is continuable at $x$ is extendible at $x$.

Proof. That (i) implies (ii) is easy. If $\mathscr{F}$ is continuable at $x$, let $D$ be an open disk about $x$ such that every function in $\mathscr{F}$ can be continued into $D$. Let $C$ be the connected component of $D \cap X$ which contains $x$. By (i), $C$ is a neighborhood of $x$ in $X$, so we can find a closed disk $D^{\prime}$ centered at $x$ such that $D^{\prime} \cap X \subset C$. If $f \in \mathscr{F}$ and we continue $f$ into $D$ and then restrict to $D^{\prime}$, the connectedness of $C$ shows that we obtain a true extension; i.e., every function in $\mathscr{F}$ extends to $X \cup D^{\prime}$.

In order to establish the converse, suppose that $X$ is not locally connected at $x$. Then there is a closed disk $D$ with center $x$ for which the component of $D \cap X$ which contains $x$ (call it $K$ ) is not a neighborhood of $x$ in $X$. For each $n$, let $U_{n}$ be an open connected set in $C$ which contains $K$, whose boundary does not intersect $D \cap X$, and no point of which is further than $1 / n$ from $K$; we may also choose $U_{n}$ so that it does not contain $D \cap X$. Let $V_{n}$ be an open subset of $C-\bar{U}_{n}$ which contains $(D \cap X)-\left(U_{n} \cap X\right)$, and such that each component of $V_{n}$ meets $D \cap X$. Set $W_{n}=(C-D) \cup U_{n} \cup V_{n}$. Let $\Delta$ be the interior of $D$ and set

$$
S_{n}=(\Delta \times\{0\}) \cup\left(W_{n} \times\{1\}\right) .
$$

Let $\widetilde{S}_{n}$ be the quotient space of $S_{n}$ by the equivalence relation which identifies $(z, 0)$ with $(z, 1)$ for each $z$ in $U_{n} \cap \Delta$. There is a continuous map $\varphi: X \rightarrow \widetilde{S}_{n}$ which sends the point $x$ to the equivalence class of $(x, 1)$, and a natural map $\psi: \widetilde{S}_{n} \rightarrow C$ that sends the class of $(z, \alpha)$ to $z$ (whether $a=0$ or $a=1$ ). Evidently $\psi$ is a local homeomorphism and induces on $\widetilde{S}_{n}$ the structure of an open Riemann surface without branch points. Note that $\psi \circ \varphi=$ identity, so there is an open neighborhood $Q$ of $\varphi(X)$ in $\widetilde{S}_{n}$ such that $\psi \mid Q$ is a homeomorphism.

Since $K$ is not a neighborhood of $x$ in $X$, for each integer $k$ we can find an integer $n$ and a point $t_{k}$ in $\Delta \cap V_{n}$ whose distance to $x$ does not exceed $1 / k$. Let $p$ be the equivalence class of $\left(t_{k}, 0\right)$ in $\widetilde{S}_{n}$ and let $q$ be the equivalence class of $\left(t_{k}, 1\right)$. Since $p \neq q$ and $\widetilde{S}_{n}$ is an open Riemann surface, we can find an analytic function $h_{k}$ on $\widetilde{S}_{n}$ for which $h_{k}(p) \neq h_{k}(q)$. Set $f_{k}=h_{k} \circ(\psi \mid Q)^{-1}$; then $f_{k}$ is analytic near $X$ and has no extension to $X \cup\{z: \operatorname{dist}(z, x) \leqq 1 / k\}$. On the other hand, if $\widetilde{J}$ is the image of $\Delta$ in $\widetilde{S}_{n}$, then $\widetilde{f}_{k}=h_{k} \circ(\psi \mid \widetilde{J})^{-1}$ is a continuation of $f_{k}$ into $\Delta$. Hence the family $\left\{f_{k}\right\}$ is continuable at $x$ but not extendible at $x$. 
We remark that, if $X$ is a compact subset of $C^{m}(m \geqq 2)$, then Theorem 5 no longer holds. There is of course no difficulty in showing that (i) implies (ii), but use of Hartog's theorem provides easy examples to show that (ii) does dot imply (i). It seems possible that a result analogous to Theorem 5 could be proved if we replaced $X$ by its "envelope of holomorphy" (see [8]). This might be quite difficult, however, since envelopes of holomorphy of compact sets in $C^{m}$ can be extremely badly behaved (see [13]).

\section{REFERENCES}

1. L. V. Ahlfors and L. Sario, Riemann Surfaces, Princeton University Press, Princeton, N. J., 1960.

2. G. R. Allan, H. G. Dales and J. P. McClure, Pseudo-Banach algebras, Studia Math., 40 (1971), 55-69.

3. F. T. Birtel, Algebras of analytic functions, Tulane University Lecture Notes, New Orleans, La., 1973.

4. K. Borsuk, Shape Theory, Aarhus University Lecture Notes, Aarhus, Denmark, 1971.

5. R. E. Edwards, Functional Analysis; Theory and Applications, Holt, Rinehart and Winston, New York, 1965.

6. M. Greenberg, Lectures on Algebraic Topology, W. A. Benjamin, New York, 1967.

7. R. C. Gunning and H. Rossi, Analytic Functions of Several Complex Variables, Prentice-Hall, Englewood Cliffs, N. J., 1965.

8. F. R. Harvey and R. O. Wells, Jr., Compact holomorphically convex subsets of a Stein manifold, Trans. Amer. Math. Soc., 136 (1969), 509-516.

9. L. van Hove, Topologie des espaces fonctionelles et des groupes infinis de transformations, Acad. Roy, Belgique Bull. Cl. Sci., (5) 38 (1952),333-351.

10. H. Komatsu, Projective and injective limits of weakly compact sequences of locally convex spaces, J. Math. Soc. of Japan, 19 (1967), 366-383.

11. G. T. Whyburn, Analytic topology, American Mathematical Society, New York, 1942.

12. W. R. Zame, Algebras of analytic germs, Trans. Amer. Math. Soc., 174 (1972), 275-288.

13. W. R. Zame, Analytic structure in some analytic function algebras, Trans. Amer. Math. Soc., 203 (1975), 215-226.

Received August 22, 1974. Supported in part by National Science Foundation Grants PO 37961 and PO 37961-001.

State University of New YoRK 


\section{PACIFIC JOURNAL OF MATHEMATICS}

\section{EDITORS}

RICHARD ARENS (Managing Editor)

University of California

Los Angeles, California 90024

\section{J. DugundJI}

Department of Mathematics University of Southern California Los Angeles, California 90007

D. Gilbarg and J. Milgram

Stanford University

Stanford, California 94305
University of Washington Seattle, Washington 98105

\section{ASSOCIATE EDITORS}
E. F. BECKENBACH
B. H. NeumanN
F. WolF
K. YoShIDA

\section{SUPPORTING INSTITUTIONS}

\author{
UNIVERSITY OF SOUTHERN CALIFORNIA \\ STANFORD UNIVERSITY \\ UNIVERSITY OF TOKYO \\ UNIVERSITY OF UTAH \\ WASHINGTON STATE UNIVERSITY \\ UNIVERSITY OF WASHINGTON \\ $\stackrel{*}{*} \stackrel{*}{*} \stackrel{*}{ }{ }^{*}$ AMERICAN MATHEMATICAL SOCIETY
}

The Supporting Institutions listed above contribute to the cost of publication of this Journal, but they are not owners or publishers and have no responsibility for its content or policies.

Mathematical papers intended for publication in the Pacific Journal of Mathematics should be in typed form or offset-reproduced, (not dittoed), double spaced with large margins. Underline Greek letters in red, German in green, and script in blue. The first paragraph or two must be capable of being used separately as a synopsis of the entire paper. Items of the bibliography should not be cited there unless absolutely necessary, in which case they must be identified by author and Journal, rather than by item number. Manuscripts, in triplicate, may be sent to any one of the editors. Please classify according to the scheme of Math. Reviews, Index to Vol. 39. All other communications should be addressed to the managing editor, or Elaine Barth, University of California, Los Angeles, California, 90024.

The Pacific Journal of Mathematics expects the author's institution to pay page charges, and reserves the right to delay publication for nonpayment of charges in case of financial emergency.

100 reprints are provided free for each article, only if page charges have been substantially paid. Additional copies may be obtained at cost in multiples of 50 .

The Pacific Journal of Mathematics is issued monthly as of January 1966. Regular subscription rate: $\$ 72.00$ a year (6 Vols., 12 issues). Special rate: $\$ 36.00$ a year to individual members of supporting institutions.

Subscriptions, orders for back numbers, and changes of address should be sent to Pacific Journal of Mathematics, 103 Highland Boulevard, Berkeley, California, 94708.

\section{PUBLISHED BY PACIFIC JOURNAL OF MATHEMATICS, A NON-PROFIT CORPORATION}

Printed at Kokusai Bunken Insatsusha (International Academic Printing Co., Ltd.), 270, 3-chome Totsuka-cho, Shinjuku-ku, Tokyo 160, Japan.

\section{Copyright (C) 1975 by Pacific Journal of Mathematics} Manufactured and first issued in Japan 


\section{Pacific Journal of Mathematics}

\section{Vol. 57, No. $2 \quad$ February, 1975}

Norman Larrabee Alling, On Cauchy's theorem for real algebraic curves with boundary .......

Daniel D. Anderson, A remark on the lattice of ideals of a Prüfer domain ..................

Dennis Neal Barr and Peter D. Miletta, A necessary and sufficient condition for uniqueness of

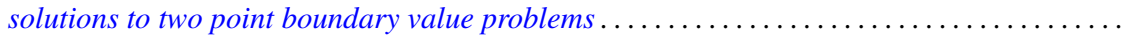

Ladislav Beran, On solvability of generalized orthomodular lattices . . . . . . . . . . ........

L. Carlitz, A three-term relation for some sums related to Dedekind sums . . . . . . . . . .....

Arthur Herbert Copeland, Jr. and Albert Oscar Shar, Images and pre-images of localization

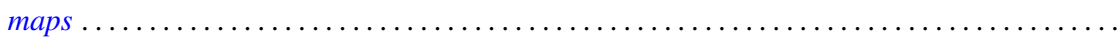

G. G. Dandapat, John L. Hunsucker and Carl Pomerance, Some new results on odd perfect

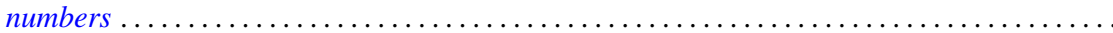

M. Edelstein and L. Keener, Characterizations of infinite-dimensional and nonreflexive

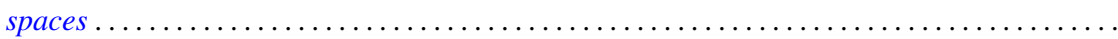

Francis James Flanigan, On Levi factors of derivation algebras and the radical embedding

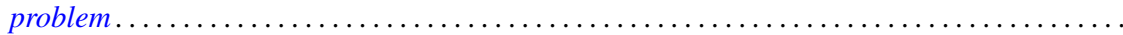

Harvey Friedman, Provable equality in primitive recursive arithmetic with and without

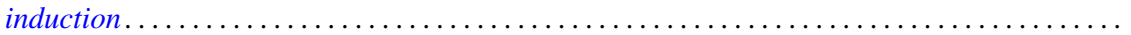

Joseph Braucher Fugate and Lee K. Mohler, The fixed point property for tree-like continua with

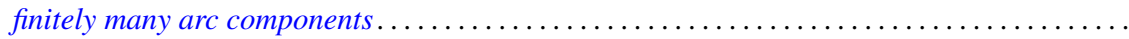

John Norman Ginsburg and Victor Harold Saks, Some applications of ultrafilters in

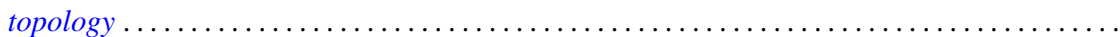

Arjun K. Gupta, Generalisation of a "square" functional equation .....................

Thomas Lee Hayden and Frank Jones Massey, Nonlinear holomorphic semigroups ..........

V. Kannan and Thekkedath Thrivikraman, Lattices of Hausdorff compactifications of a locally

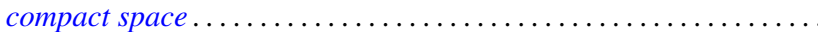

J. E. Kerlin and Wilfred Dennis Pepe, Norm decreasing homomorphisms between group

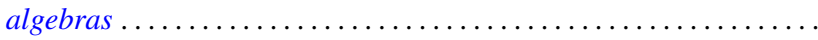

Young K. Kwon, Behavior of $\Phi$-bounded harmonic functions at the Wiener boundary ...

Richard Arthur Levaro, Projective quasi-coherent sheaves of modules .

Chung Lin, Rearranging Fourier transforms on groups...........................

David Lowell Lovelady, An asymptotic analysis of an odd order linear differential equation . . 4475

Jerry Malzan, On groups with a single involution .......................... 481

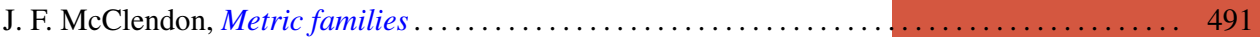

Carl Pomerance, On multiply perfect numbers with a special property .

Mohan S. Putcha and Adil Mohamed Yaqub, Polynomial constraints for finiteness of semisimple rings. .

Calvin R. Putnam, Hyponormal contractions and strong power convergence . . . . . . . . . 531

Douglas Conner Ravenel, Multiplicative operations in $\mathrm{BP} * \mathrm{BP} \ldots \ldots \ldots \ldots \ldots \ldots \ldots \ldots \ldots .539$

Judith Roitman, Attaining the spread at cardinals which are not strong limits . . . . . . . . . 545

Kazuyuki Saitô, Groups of *-automorphisms and invariant maps of von Neumann algebras . . . 553

Brian Kirkwood Schmidt, Homotopy invariance of contravariant functors acting on smooth

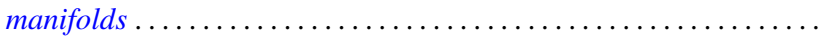

Kenneth Barry Stolarsky, The sum of the distances to $N$ points on a sphere.

Mark Lawrence Teply, Semiprime rings with the singular splitting property.

J. Pelham Thomas, Maximal connected Hausdorff spaces..............

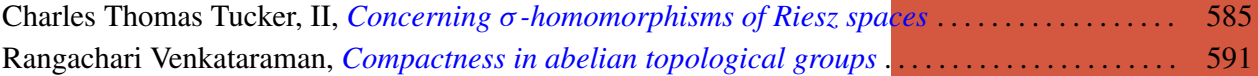

William Charles Waterhouse, Basically bounded functors and flat sheaves . . . . . . . . . . . 597

David Westreich, Bifurcation of operator equations with unbounded linearized part ......... 611

William Robin Zame, Extendibility, boundedness and sequential convergence in spaces of 